\title{
Magic, Bayes and wows: a Bayesian account of magic tricks
}

\author{
Pablo Rodrigo Grassi ${ }^{1,2,3,{ }^{*}}$ and Andreas Bartels ${ }^{1,2,3}$ \\ ${ }^{1}$ Department of Psychology, University of Tübingen, Germany \\ ${ }^{2}$ Centre for Integrative Neurosciences, Tübingen, Germany \\ ${ }^{3}$ Max-Planck Institute for Biological Cybernetics, Tübingen, Germany \\ * Corresponding author: \\ pablo.grassi@cin.uni-tuebingen.de
}

\begin{abstract}
Magic tricks have enjoyed an increasing interest by scientists. However, most research in magic focused on isolated aspects of it and a conceptual understanding of magic, encompassing its distinct components and varieties, is missing. Here, we present an account of magic within the theory of Bayesian predictive coding. We present the "wow" effect of magic as an increase in surprise evoked by the prediction error between expected and observed sensory data. We take into account prior knowledge of the observer, attention, and (mis-)direction of perception and beliefs by the magician to bias the observer's predictions and present two examples for the modelling of the evoked surprise. The role of misdirection is described as everything that aims to maximize the surprise a trick evokes by the generation of novel beliefs, the exploitation of background knowledge and attentional control of the incoming information. Understanding magic within Bayesian predictive coding allows unifying all aspects of magic tricks within one framework, making it tractable, comparable and unifiable with other models in psychology and neuroscience.
\end{abstract}

Keywords: Magic, Bayesian surprise, predictive coding, misdirection, attention 


\section{Introduction}

Magic is about violating expectations: a freely selected card is guessed from a random deck, a coin magically appears in a hand, a chair levitates on stage. We are surprised, because our expectations are suddenly questioned by our senses. This makes magic fascinating for the viewer and also for the researcher.

The effect magic evokes has been described as an extraordinary phenomenological experience, namely, as the "sense of wonder induced by seeing an apparently impossible event" (Rensink and Kuhn, 2015a). This experience of wonder and "impossibility" is induced by the profound mismatch between memory-based expectations and a sensory occurrence (Smith et al., 2016).

Notably, it is exactly this - the prediction error between expected and actual sensory information - that has increasingly become one of central elements of recent developments in cognitive neuroscience: Bayesian predictive coding theories of brain function (Mumford, 1992; Rao and Ballard, 1999; Friston and Kiebel, 2009; Clark, 2013). Bayesian theories of brain function understand the brain as an inference organ that aims to minimize the prediction error between expected and actual sensory information. Magic, in turn, seems to exploit these prediction errors to maximize surprise. Intuitively, it appears promising to frame magic and misdirection within this influential theory of brain function and describe its effect as an increase of prediction error or surprise.

However, although there has been an increasing effort in propagating the scientific investigation of magic using the tools of cognitive science (see Kuhn et al., 2008; Macknik et al., 2008; Rensink and Kuhn, 2015a; Thomas et al., 2015; Quiroga, 2016; Garcia-pelegrin et al., 2020 for an overview), the connection of magic research to Bayesian predictive coding and cognitive neuroscience in general is still thin.

In particular, most studies of magic focused their analyses on behavioural responses and eye movements (e.g. Kuhn and Tatler, 2004; Kuhn and Land, 2006; Cui et al., 2011; Barnhart and Goldinger, 2014; Mohr et al., 2015; Ekroll et al., 2016). The link to neural signals has been largely neglected. Only three studies used neural signals to study our response to magic: two closely-related functional magnetic resonance imaging (fMRI) studies localized brain areas involved in the perception of magic (Parris et al., 2009; Danek et al., 2015), while one electroencephalogram (EEG) study focused in the evoked potential related to a "magical" event (Caffaratti et al., 2016).

The purpose of this paper is to close this gap. We aim to integrate the study of magic with cognitive neuroscience using an account of magic within the context of Bayesian predictive coding. The use of this established framework can help linking the study of magic to cognitive neuroscience and to develop hypothesis-driven physiological studies.

The relation of magic to Bayesian predictive coding is not new (see e.g. Kuhn 2019). Previous studies have related magic to Bayesian inference and predictive coding in different formulations (e.g. Barnhart, 2010; Danek et al., 2015; Van de Cruys et al., 2015; Kuhn and Rensink, 2016). However, these references were usually given as a possible interpretation of certain magic effects and as an interesting note of something worth delving deep into.

We will proceed as follows: in a first section, we shortly introduce Bayesian predictive coding and describe the Bayesian brain's aim of minimization of prediction error and surprise. Then, we will show how magic effects can be understood as an increase of (Bayesian) surprise resulting from the mismatch between expectations and sensory information. We exemplify its computation using Bayesian models applied to two simple magic tricks. Thereafter, we describe misdirection as all elements of the preparation phase that aim to maximize the surprise, and we present three types of misdirection based on their effects on Bayesian inference. Finally, we argue that the understanding of experience of magic as surprise within a Bayesian predictive coding framework enables a variety of research questions that can be addressed using magic tricks as stimuli exploiting known neural and behavioural responses.

\section{Bayesian predictive coding}

A key function of our sensory and cognitive systems is to provide us with trustworthy representations about physical properties of the world in order to allow for a meaningful interaction with it. Yet, the sensory information is incomplete, noisy, and ambiguous, making it 
an incredibly complex task to derive the underlying physical sources from it. As an example, the whole process of transforming the visual input from a scene received in both our retinas back again into a representation of the scene consisting of segregated elements is an sophisticated achievement of our visual system - but also one thought to require more than a third of the human brain (Felleman and Van Essen, 1991). For any given retinal signal there is an infinite number of possible sources that could have caused it (Kersten and Yuille, 2003). To cope with this fundamental ambiguity of perception our brain employs prior knowledge about the world based on experienced statistical regularities to predict the most likely causes of the incoming information.

Recent cognitive theories of brain function understand the brain as a probabilistic inference system that attempts to explain the input it receives by generating hypotheses of the possible causes of it by means of Bayesian inference (Kersten et al., 2004; Friston, 2005; Friston and Kiebel, 2009; Clark, 2013). Bayesian inference involves the combination of observed information (likelihood) with probabilistic predictions based on previous knowledge (prior beliefs) to calculate a posterior probability, which corresponds to the most likely interpretation. This inference process has been hypothesized to be implemented in the brain through predictive coding (Mumford, 1992; Rao and Ballard, 1999). Bayesian predictive coding theories understand the brain as a hierarchical system whose aim is to maximize the evidence for its probabilistic model of the world by minimizing the difference between its predictions (i.e. prior beliefs) with the sensory data. The difference between prior beliefs and incoming sensory information is referred to as prediction error, which, in short, reports the "surprise" induced by the mismatch. If the predictions fail to explain the incoming information, then the prediction error gives novel information to update the probabilistic beliefs about the world and is therefore the fundamental basis of learning (Schultz et al., 1997; Friston, 2005; Friston and Kiebel, 2009).

In sum, following this account, a key task of the brain is to acquire knowledge and build a world model, such that prediction errors between its probabilistic world model and actual observations are minimized ("not to be surprised"). However, this very process, updating and improving the model, and adapting it throughout the dynamics of human life, necessitates seeking out the unexpected and unknown, and thus, being surprised (Schwartenbeck et al., 2013; Clark, 2018). Accordingly, curiosity-driven learning and noveltyseeking explorative human behaviour necessarily accompanies the Bayesian predictive coding imperative of "minimize long-term prediction error". This essential aspect of human behaviour is expressed in the practices of, just to name a few, literature, humour, games, puzzles, visual arts and, relevant for us, magic.

In the following, we will present three main features of Bayesian predictive coding, which are important for the understanding of magic: its hierarchical structure, contextual dependency and attentional modulation.

\subsection{Inference: across the cortical hierarchy}

In Bayesian predictive coding, the minimization of prediction error is hypothesized to occur concurrently across different levels of the cortical hierarchy, which are interconnected via feedforward and feedback signalling pathways. While prediction errors are fed forward to the next higher level of the cortex to update our beliefs about of the world, predictions are sent back to lower-tier areas via recurrent connections to be compared with the incoming information (Mumford, 1992; Rao and Ballard, 1999; Friston, 2005). Given the functional specialization of the cortex, the content of prediction errors differs largely depending on the areas along the cortical hierarchy in which they are generated. Mismatch between a particular predicted motion and the incoherent motion can generate prediction error responses in the motion processing area hMT+ (e.g. Harrison et al., 2007), mismatch between an expected tone and the heard one will show prediction error responses in the primary auditory cortex (e.g. Todorovic et al., 2011), unexpected faces will increase responses in the fusiform face area (e.g. Egner et al., 2010), and so forth. Prediction errors thus convey a manifold of surprise signals that map onto distinct regions and help the brain update its representation of the causal structure of the world at different sensory and cognitive levels. 


\subsection{Contextual modulation: optimizing predictions}

Our expectations are strongly modulated by contextual information. The occurrence of an elephant in the streets of a German town would be surprising, while it would be expected in a zoo. Similarly, we are perhaps surprised when seeing David Copperfield fly across the stage but expect this of astronaut Chris Hadfield in the international space station. Clearly, every context evokes an extensive set of individual probabilities at all levels of perception and cognition of what one is to expect in a particular situation via memory-based associations, statistical regularities and general knowledge of the world. Contextual cues can aid the selection of priors in a particular situation and therefore strongly affect expectations and, consequently, our surprise. In the visual domain, for example, it is known that contextual information strongly affects processing of visual perception (Chun, 2000; Todorović, 2010) and the main function of "gist" perception of a scene is probably to aid the fast classification of objects in it (Kveraga et al., 2007; Bar, 2009).

\subsection{Attentional modulation: optimizing the precision of prediction errors}

Following a plane crash, a huge effort is spent to pinpoint exactly what went wrong - precise knowledge of faults is required in order to predict and prevent future failures. Similarly, the quality with which our inferential system can update its world model depends on the precision (inverse of the variance) of the prediction error. Precision is high when both, predictions as well as sensory input, are precise. Accordingly, it has been suggested that prediction errors are weighted by their precision and that the brain therefore also needs to estimate this precision. It has been proposed that the internal process mediating the optimization of the precision estimates is attention (Friston and Kiebel, 2009; Hohwy, 2012). Attentional processes enhance the precision of specific prediction errors related to current expectations, thereby increasing their relative weight during inference and learning. Put simply, we have higher trust in the prediction errors of information we are paying attention to. For example, it is hypothesized that spatial attention to a particular location of the visual field increases the weight that is put on the information of corresponding sensory neurons (prediction error units) in the visual cortex representing that portion of the visual field and decrease the weight given to non-attended non-corresponding units (Hohwy, 2012).

\section{Magic tricks and Bayesian inference}

In this second section we present an understanding of magic within the above-sketched Bayesian predictive coding framework. First, we briefly describe the basic structure of a magic trick and its separation into the preparation phase and the effect. Then, we will show that the effect of wonder evoked by magic can be understood as the experience of surprise evoked by the sudden increase of prediction error due to a profound mismatch between memory-based prior beliefs and sensory observations. Thereafter, we will illustrate how magicians use methods of misdirection to construct this mismatch during the preparation phase to maximize surprise.

\subsection{The structure of a magic trick}

Magic tricks are usually described as consisting of two distinct phases: a preparation phase (also containing its methods) and a final effect (Lamont and Wiseman, 1999; Rensink and Kuhn, 2015a; Smith et al., 2016). The effect of a magic trick is characterized by the sudden phenomenological experience of wonder that it evokes, which can have different contents (e.g. a coin can appear, disappear, multiply, change, etc.). We refer to this experience as the "wow" effect, which we will describe as surprise. The method is basically how the trick works (e.g. mechanical devices, sleight of hand, visual illusions, etc.). The preparation phase is the whole sequence of events and actions that are presented to the spectators (e.g. showing the objects, explaining the procedure, shuffling the cards, etc.), which culminates in the 
experience of wonder or the "wow" effect. ${ }^{1}$ The main purpose of the preparation phase is to allow for the effect (Lamont and Wiseman, 1999). Thus, the creation of a magical event is necessarily dependent on all of the preceding events and, most importantly, on the whole history of believed states by the observers (cf. Lamont and Wiseman, 1999; Smith et al., 2016).

Imagine, for example, the following coin production trick: A magician shows a seemingly empty hand. The hand is closed and opened again to surprisingly reveal a coin inside. The perception of a coin in an opened hand is only perceived as something "magical" if and only if this coin was not supposed to be there, and because we know that objects do not arbitrarily appear in people's hands. Only given these prior beliefs in the viewers mind is the occurrence of the coin perceived as something surprising.

\subsection{The "wow" effect of magic as surprise}

In the introduction, we described how the experience of magic is often defined as the emotion evoked by an expectation violation in which something "impossible" occurs (i.e. the "wow" effect). Crucially, in a Bayesian framework this corresponds to what we experience in view of the occurrence of an extremely unexpected event (with a prior probability close to 0 ). Given that unexpected events are only so in the light of background beliefs, we can describe the "wow" effect created by magic tricks as the phenomenological experience resulting from a profound mismatch (prediction error) between these memory-based beliefs (priors) and the incoming sensory information (likelihood). The emotion arising from such a mismatch is usually referred to as surprise (Barto et al., 2013). We can accordingly describe the "wow" effect of magic as resulting from a peak in prediction error and, in consequence, as surprise.

Consider the simple coin production example given above: the viewer believes that the magicians' hands are empty (prior belief) and knows that objects do not arbitrarily appear (hyperprior), so that seeing a coin in the opening hand (sensory data) elicits a large prediction error (mismatch between priors and sensory data) that is experienced as surprise.

It is important to note that the Bayesian view is always a probabilistic and not a binary one. Bayesian views represent degrees of beliefs as probability distributions that are the result from inductive learning of statistical regularities. Hence, the almost canonical understanding of magic effects as the experience of "impossibility" (Rensink and Kuhn, 2015a; Smith et al., 2016; Lamont, 2017) $)^{2}$ has, in the Bayesian view, to be rephrased with "highly unlikely". This is of relevance from a theoretical and technical perspective: the Bayesian framework takes the universal and inherent uncertainty of our internal world-model into account. This is also of technical importance as probability calculations rely on non-zero values.

Observers of magic might indeed describe a specific occurrence as "impossible", but this just reflects that a specific belief with a high degree of certainty was shown to be false. Clearly, a magician guessing a card out of a deck of cards is not impossible but just improbable, and yet, magic.

Moreover, it is noteworthy that while not all surprises are experienced as magic, all magic is experienced as surprise. Hence, magic can be viewed as a subset and special form of surprise. This form of surprise could be characterized by its strong intensity, positive valence and the kind of violated expectations it arises from (i.e. laws of physics, causality, etc.). The lack of immediately available explanation may also contribute. Yet, it may prove difficult to base a definition of the effect of magic entirely on these grounds.

\footnotetext{
${ }^{1}$ This basic structure can also be hierarchically organized into more complex magic routines that consist of a series of different effects and their intertwined preparation phases, which can culminate into an even larger "wow" effect. A common principle in magic routines is to use a first magic effect (e.g. guessing a card) to generate a false expectation about a subsequent effect (e.g. all cards change color). In such cases, the initial effects are elements of misdirection, which create misleading expectations of the later ones.

2 The effect of magic is often described to arise from a "contradiction", "conflict" or "dilemma" between beliefs: "The response to a magic trick is the response to a seemingly impossible event. [...] It is this juxtaposition between ' $x$ cannot happen' and ' $x$ happens' that provokes the experience of magic." (Lamont, 2017), “... impossibility exists as a contradiction between a perception-supported belief [...] and a memory-supported belief [...]" (Smith et al., 2016), "[...] magic creates a cognitive conflict between things we experience and things we believe to be impossible" (Kuhn, 2019).
} 
Potentially a key aspect of surprise experienced in magic performance is that observers' attention is fully engaged on the items of interest - indeed, it is a core aspect of a good magician to fortify a prior belief (e.g. "there is no coin in the hand") and make observers pay attention to this. Both aspects consequently enhance the prediction error and surprise. In contrast, many real-life surprises (such as finding a coin on the street) are coincidental, with less or no explicit attention directed to the street, and comparably weak prior beliefs about finding anything on the street. Also, immediate explanations are available (e.g. someone lost the coin).

Finally, please note that the fact that the audience of a magic show generally expects to be surprised or, in context of a particular trick, expects something specific to happen, does not pose a problem to the understanding of the effect of magic as surprise. These kind of vague expectations of magic (usually) do not prevent bringing the audience into the right mind-set to experience surprise, as magicians are well-trained to deal with them and can even use them to their advantage.

In sum, we propose the "wow" effect of magic to be understood as surprise and the magician's aim described as: to evoke surprise. In consequence, if we understand that a key element of the brain is to seek novelty and surprises in order to improve its world model, then magic tricks satisfy exactly this central need: to maximize the prediction error by minimizing the evidence supporting prior beliefs ("to evoke surprise").

\subsection{Neural evidence for the "wow" effect as surprise}

We are aware of only three previous studies that investigated neural responses to the experience of magic tricks. The results of all three provide neural evidence that supports the notion of a relatedness between the "wow" effect as surprise signal and Bayesian predictive coding (Parris et al., 2009; Danek et al., 2015; Caffaratti et al., 2016).

Both fMRI studies were similar: brain responses to videos showing magic tricks (e.g. making a coin disappear) were compared to neural signals of control videos showing similar actions but no magic (e.g. showing a coin) by means of a standard univariate approach (Parris et al., 2009; Danek et al., 2015). Crucially, participants in both studies reported the magic videos to be significantly more surprising that the control videos. They revealed consistent increased activation in the medial and dorsolateral prefrontal cortex (mPFC and dIPFC) and dorsal anterior cingulate cortex (dACC) during the perception of magic. Moreover, Danek et al., (2015) revealed the differential involvement of further areas, including the ventral striatum and clusters along the ventral visual stream. Together, these results are consistent with a plethora of studies that show the involvement of the very same areas in the predictive processing of unexpected and surprising information. The MPFC and specifically the dACC have been shown to be involved in the detection of discrepancies between actual and predicted high-level action outcomes (Alexander and Brown, 2011, 2019) and, more generally, in the processing of surprise (Ide et al., 2013; Schwartenbeck et al., 2016), while the dIPFC and ventral striatum show strong prediction error responses to expectation violations of learned causal associations (Fletcher et al., 2001; Corlett et al., 2004; Turner et al., 2004). Finally, it is known that feature-selective visual areas signal lower-level prediction error when presented with unexpected visual stimuli (e.g. Summerfield et al., 2008; Mayrhauser et al., 2014; Utzerath et al., 2017).

Accordingly, the EEG study revealed differential P300 evoked potentials during repeated viewing of a magic trick compared to control conditions (Caffaratti et al., 2016). The observed P300 responses are known to be evoked during odd-ball paradigms, i.e. novel and surprising stimuli (Linden, 2005; Polich, 2007) and have been recently hypothesized to reflect prediction error and surprise (Mars et al., 2008; Wacongne et al., 2011; Kolossa et al., 2012; Kopp et al., 2016).

\subsection{Computing the "wow" effect as Bayesian surprise}

One advantage of understanding magic in a Bayesian framework is that it enables the cognitive modelling and quantification of its effect. To do this, we will use the theory of Bayesian surprise presented by Itti and Baldi, which provides a formal and quantitative theory of surprise in Bayesian terms (Itti and Baldi, 2005, 2009; Baldi and Itti, 2010). In their approach, subjective beliefs (or internal world models, $M$ ) are understood as probabilities 
$(P(M))$ that are updated into posterior beliefs $P(M \mid D)$ as data $D$ is acquired using Bayes theorem (see Figure 1A). They describe any existing subjective belief as a prior probability distribution $\{P(M)\}_{M \in \mathcal{M}}$ over models in a model space $\mathcal{M}$. An example of such a background belief could be something like $P(M)=$ "the hands of the magician are empty". In view of new data $D$ (e.g. "empty hands") the subjects update their prior distribution $P(M)$ (i.e. background belief or prior probability) into a posterior distribution $P(M \mid D)=$ "the hands are indeed empty" via Bayes theorem:

$$
\forall M \in \mathcal{M}, \quad P(M \mid D)=\frac{P(D \mid M)}{P(D)} P(M)
$$

The new data $D$ (e.g. "empty hands") carries no surprise if it does not change the belief of the observer ("the hands are indeed empty"). In contrast, the observed data $D$ (e.g. "a coin") is surprising if the posterior distribution $P(M \mid D)$ differs from the prior distribution $P(M)$ ("Wow! Where does this coin come from??"). Itti and Baldi suggest quantifying "surprise" as the difference between the posterior and prior distributions. They suggest doing so using the socalled Kullback-Leibler divergence:

$$
S(D, \mathcal{M})=K L(P(M \mid D), P(M))=\int_{\mathcal{M}} P(M \mid D) \log _{2} \frac{P(M \mid D)}{P(M)} d M
$$

Itti and Baldi define the unit of surprise for a single model as a "wow". This corresponds to a two-fold variation between the posterior distribution $P(M \mid D)$ and prior distribution $P(M)$ i.e. as $\log _{2} P(M \mid D) / P(M)$. The total number of wows in view of new data $D$ is given by the integration over all models in space $\mathcal{M}$. Thus, according to this approach, surprise is a measure of the difference between beliefs before and after an observation, and surprising events are those that are not well predicted by the viewers' prior beliefs. To exemplify the computation of Bayesian surprise and its application to study of magic, let us apply it to two toy examples.

\subsubsection{A simple card trick}

Imagine the following classic situation of card magic: a magician guesses a card that was freely selected by a viewer from a deck of 52 cards. We assume that the magician effectively hid the secret sleight of hand. We further assume that the viewers' prior belief at the time point of the magic effect is that it is extremely unlikely that the magician selects the correct card (close to 1/52). To the viewers' surprise, the magician guessed the card repeatedly.

To quantify the amount of surprise this trick evokes, we can think of the trick as a coin toss with an unfair coin (binary data that can either be a success or a failure and can be described as a Bernoulli distribution), and the viewers' belief as trying to judge how probable a success is. This model is parameterized by the viewer's estimate of the magician's skills, which is simply the probability $0 \leq w \leq 1$ of the magician picking the correct card (see Figure 1B left for a graphic of the model). A bad magician will have a low $w$, while a good magician will have a high $w$. For convenience, we will model the viewer's updating of their beliefs (of the magician's skill) and their surprise analytically by using conjugate priors. Conjugate priors are prior distributions $P(M)$, that when multiplied with a specific type of likelihood function $P(D \mid M)$ during Bayesian updating $(P(M \mid D) \propto P(D \mid M) P(M))$, generate a posterior distribution $P(M \mid D)$ that is of the same family as the prior distribution. The Beta distribution with the parameters $\alpha$ and $\beta$ is the conjugate prior for binomial and Bernoulli likelihood distributions. We will hence use the Beta distribution as conjugate prior $P(M)$ for the Bernoulli likelihood distribution of card picks $P(D \mid M)$. The posterior distribution $P(M \mid D)$ is accordingly also a Beta distribution that can now be computed easily: the parameters of the posterior Beta distribution $P(M \mid D)$ are $\alpha^{\prime}=\alpha+n$ and $\beta^{\prime}=\beta+(N-n)$, where $n$ is the number of correct picks and $N$ the number of tricks. For example, a prior Beta distribution with parameters $\alpha=1$ and $\beta=1$ will have, after two successful guesses out of five guesses $(n=2, N=5)$, a posterior Beta distribution with $\alpha=3$ and $\beta=4$, because $\alpha$ increases with the number of correct guesses and $\beta$ increases with failed guesses.

The exact analytical measurement of surprise for a binomial model is then given by: 


$$
S(D, \mathcal{M})=\log \frac{C^{\prime}}{C}-n[\Psi(\alpha+\beta+N)-\Psi(\alpha+n)]-(N-n)[\Psi(\alpha+\beta+N)-\Psi(\beta+N-n)]
$$

where $C$ is $\Gamma(\alpha+\beta) /[\Gamma(\alpha) \Gamma(\beta)]$ ( $\Gamma$ is the gamma function) and $\Psi$ is the digamma function (for a detailed explanation of this equation please see Itti and Baldi, 2009).

In our example, the viewer believes the magician is not going to be capable of fooling them. Thus, the prior distribution $P(M)$ is centred on the ,just by chance" probability of a magician's skill of $w=\frac{1}{52} \approx 0.02$. However, this estimate is not going to be that precise because not all viewers know the number of cards in a deck and they also know that they are witnessing a magician, which increases their uncertainty even further. This can be mimicked in the prior Beta distribution by assuming a fairly large variance of, for example, 0.01 . Given this wanted mean $\mu$ and variance $\sigma^{2}$, we can calculate the parameters of the prior beta distribution of the observer as: $\alpha=\left(\frac{1-\mu}{\sigma^{2}}-\frac{1}{\mu}\right) \mu^{2}$ and $\beta=\alpha\left(\frac{1}{\mu}-1\right)$, which yields $\alpha \approx 0.02$ and $\beta \approx 1$ (note that the relation $\alpha / \beta$ is $1 / 50$, which has the same mean as a prior of $\alpha=1$ correct guess and $\beta=50$ failures but with a larger variance). Moreover, we assume that the magician is good, but not perfect and fails ca. 1 every 10 picks (i.e. the true probability of the magician's skills is around $w=0.9$, see Figure $1 \mathrm{~B}$ middle).

Using these parameters, we can calculate the viewer's surprise to the card trick. A failed first guess would only lead to a small surprise of 0.007 wows, because the viewer expected it. In contrast, a successful first guess evokes 2.95 wows of surprise (421 times more surprising!). If the magician successfully repeats the exact same trick, the surprise rapidly starts to go down, as the viewer's belief is now rapidly getting closer to "That's good! The magician will probably guess it again". The second time the viewer sees a successful trick it only generates a surprise of 0.2 wows (over 15 times less surprising than the first time). And after five successful repetitions it only generates 0.02 wows. The prior distribution has now strongly shifted to the right with a mean around $w=0.83$. After a larger amount of repetitions (e.g. $N=25, n=23$ ), the final posterior distribution will be close to the real magicians' skills (around 0.9) (Figure 1B right).

Imagine now a magician who adds a small twist to the trick and purposefully fails in the first two guesses (e.g. by telling the viewer that three cards will be selected and failed on guessing twice). The viewers surprise on the correct third event after seeing the magician fail twice is now 3.21 wows instead of 2.95 wows. Along the same line, if the viewer already knows the trick, or they already saw the magician perform it and only think the trick could fail by mistake, or truly believes in magic (e.g. $\alpha=9, \beta=1$ ), then a correct guess by the magician is not surprising at all ( 0.005 wows).

\subsubsection{A simple mind-reading trick}

In our second example, we go a step further and try to model the viewer's beliefs a bit more realistically. The viewer will not only be estimating the magician's skill $w$, but also two related propositional beliefs $\left(M_{1}\right.$ and $\left.M_{2}\right)$. Consider the following mind-reading trick: a magician spreads ten objects over the table and asks some viewers to freely think about one of them. The magician tells the viewers that psychic powers will be used to read their minds. The magician successfully brought all of the viewers to the belief that, in case the objects are correctly guessed, it would be because of these psychic powers (something they clearly mistrusted). Surprisingly, the magician correctly guessed the selected objects repeatedly.

How much surprise did this mind-reading trick elicit? To calculate the amount of surprise, we will now focus just on the probability of the two concurrent beliefs in the viewers mind $\left(M_{1}\right.$ and $\left.M_{2}\right)$. We will not calculate the model analytically using conjugate priors, but rather estimate the unknown variables using Markov Chain Monte Carlo sampling for computational simplicity. We assume the concurring beliefs of the observers are that either the magician has no psychic powers $\left(M_{1}\right)$ or that he has psychic powers $\left(M_{2}\right)$, which broadly correspond to the viewers belief if he is going to be successful or not $\left(P\left(M_{2}\right)=1-P\left(M_{1}\right)\right)$. Magicians with no psychic power have a probability of success of 0.1 (chance level), while magicians with psychic powers greater than 0.1 (depending on how good of a psychic they are). The number of correct guesses is $n$ out of $N$ repetitions. The probability of success is the magician's skill $w$. In case the magician has no psychic power $w=0.1$, in case he has $w=\phi$, which we assume are all uniformly distributed rates of probabilities greater than 0.1 
$(\phi \sim$ Uniform $(0.1,1))$. If the magician has psychic powers $\left(M_{1}\right)$ or not $\left(M_{2}\right)$ is determined by a binary variable $\mathrm{z}$ (with $\mathrm{z}=1$ if the magician has psychic powers and 0 if not) that will be estimated by the model. To reflect the fact that the observers do not believe that he is going to be successful (i.e. he has no psychic powers) we ascribe the variable $\mathrm{z}$ the prior probability of 0.05 ( $\mathrm{z} \sim$ Bernoulli $(0.05)$ ), which corresponds to $P\left(M_{1}\right)=0.05$ and $P\left(M_{2}\right)=1-P\left(M_{1}\right)=$ 0.95 . This informed prior reflects that the observers are more or less confidently ascribing the magician no psychic powers. Implementing these assumptions in a simple Bayesian model allows us to estimate the prior and posterior probabilities of the beliefs after $N$ repetitions (see Figure $1 \mathrm{C}$ left for a graphical depiction). In this case we compute the surprise over the two beliefs $\left(M_{1}\right.$ and $\left.M_{2}\right)$ based on the estimation of $\mathrm{z}$ as:

$$
S(D, \mathcal{M})=P\left(M_{1} \mid D\right) \log _{2} \frac{P\left(M_{1} \mid D\right)}{P\left(M_{1}\right)}+P\left(M_{2} \mid D\right) \log _{2} \frac{P\left(M_{2} \mid D\right)}{P\left(M_{2}\right)}
$$

Let us start by assuming the magician performed the trick one first time successfully. The surprise elicited is $\approx 0.26$ wows, reflecting a bit of surprise but also the possibility that the magician could have landed a lucky guess. Two successful guesses elicit already a surprise of $\approx 2$ wows and performing the trick three consecutive times successfully elicits a surprise of $\approx 3.6$ wows. The posterior belief after seeing the magician successfully perform the trick three times in a row is now close to $1(\mathrm{z} \approx 0.93)$, suggesting that the viewers are already ascribing her/him "psychic powers" or (most probably) just believe that the magician is good (Figure 1C middle). On the contrary, three fails elicit only $\approx 0.027$ wows. After just 5 successful tricks the total surprise stagnates at $\approx 4.3$ wows and stops increasing. This occurs because the viewers' beliefs reached now the probabilities $P\left(M_{1}\right) \approx 1$ and $P\left(M_{2}\right) \approx 0$, showing that they ascribed the magician "psychic" powers (which corresponds to predicting that the magician is not just guessing) and aren't surprised by the trick any more (Figure $1 \mathrm{C}$ right). 
A.
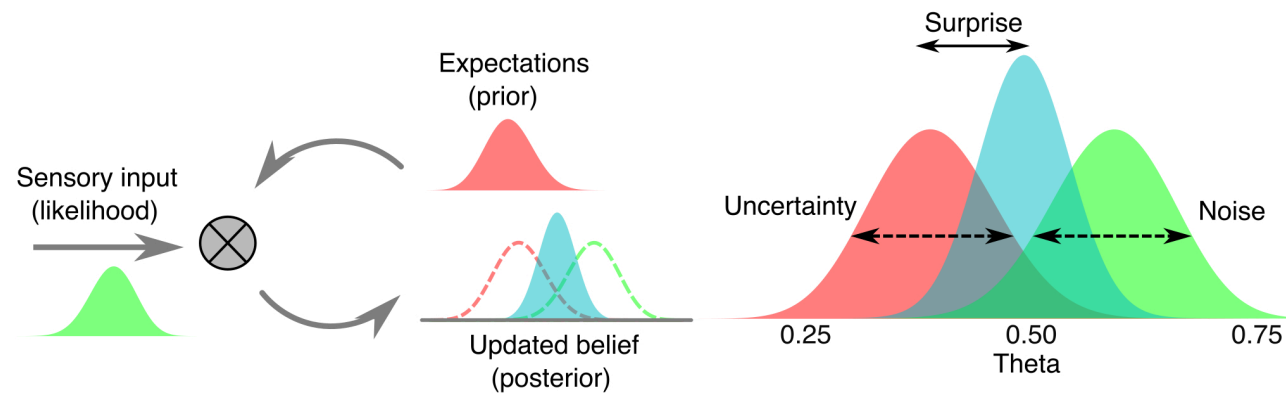

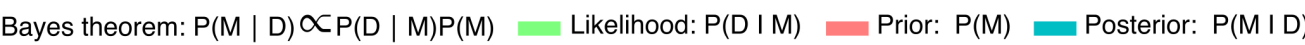

B.

Graphical model

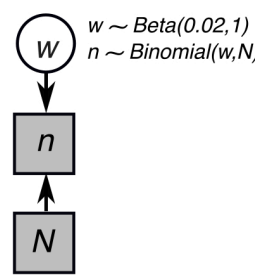

Prior and true distribution

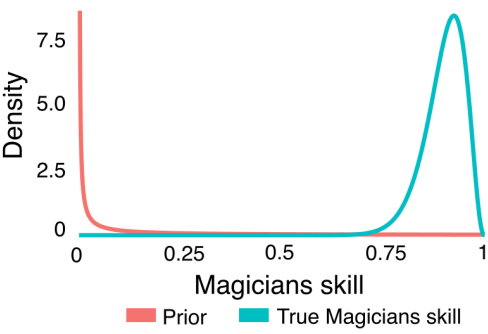

Sequential distributions

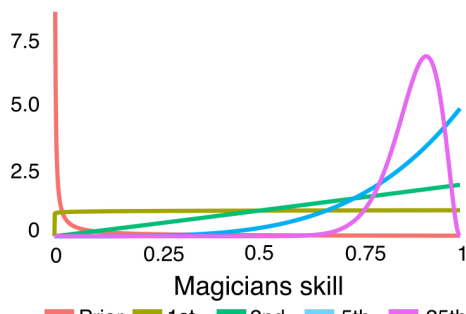

C.
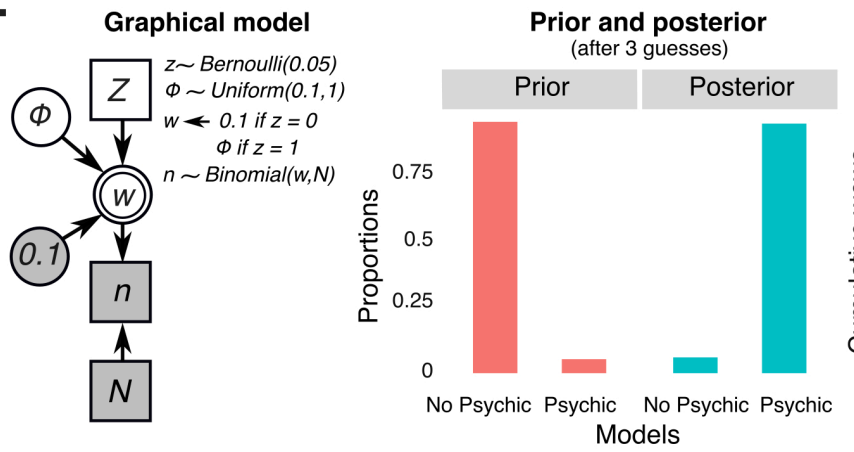

Prior and posterior

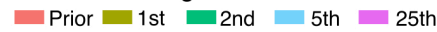

\section{Cumulative surprise}

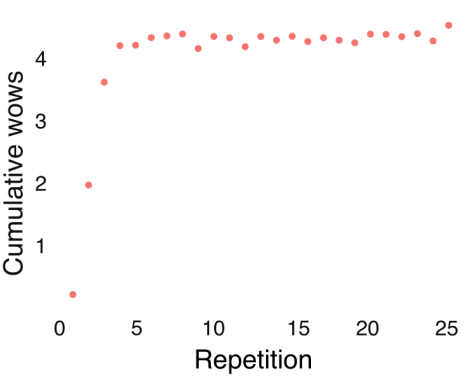

Figure 1. A. Illustration of Bayesian predictive coding. Incoming sensory data (likelihood) and expectations (priors) are represented as probability distributions (green and red, respectively). Beliefs are updated via the combination of prior and likelihood distributions following Bayes theorem (blue posterior distribution). The variance of the distributions describes the uncertainty of the priors and the noise of the sensory information. B. Left: a graphical model of the Bayesian observer of the card trick. Nodes represent the variables and arrows their dependencies. Known variables are shown grey and unknown variables are shown white. Discrete variables are represented as squared nodes, continuous variables as circular nodes. Stochastic and deterministic variables are represented as single- or double-bordered nodes, respectively. Middle: the viewer's prior beliefs and true distributions of the magician's skills. The true distribution has a mean of 0.9 and a standard deviation of 0.1 for visualization purposes. Right: prior distribution and a number of posterior distributions show how the prior belief of the viewer rapidly changes shape at the beginning and then approximates the true magicians' skills (around 0.9). The model was implemented analytically using MATLAB 2014 (https://www.mathworks.com). C. The proposed model of the psychic trick is similar to the previous one but contains an additional binary latent variable $z$, which describes the beliefs "Psychic" and "No Psychic" with the prior probabilities of 0.05 and 0.95 respectively (shown in the middle panel). With just 3 correct guesses the estimation of the variable $z$ is already close to 1 (middle panel). This rapid change is also reflected in the right plot, which shows the cumulative surprise as a function of successful repetitions. After the rapid increase in surprise, the total amount of wows reaches its maximum around 4.3 wows and stops increasing. The model was estimated in R with the JAGS library (http://mcmc-jags.sourceforge.net/) using one Markov chain with 10000 iterations and the initial parameters: $\phi=0.1$ and $z=0$ for 25 repetitions independently. For more information about the computation of Bayesian cognitive models see Lee 2008 and Lee and Wagenmakers 2013.

\subsubsection{Some remarks about the computation of surprise}

The above examples capture several intuitive aspects of the "wow" effect of magic tricks and thereby support the understanding of magic effects as surprise. They show that as the viewer realizes the magician's skills, the level of surprise drops. Also, if they believe the magician will fail, they are not surprised by failure. But in cases of divergence between expectation and outcome surprise occurs, such as when viewers expect failure of a feat that then is achieved 
by the magician. This behaviour shows that the "wow" effect of magic is directly dependent on the viewers' prior beliefs and expectations, and is in line with the intuition of how viewers experience magic. Moreover, the reduction in surprise after successful repetitions in both examples directly represents one of the long-standing rules of magic: "never do the same trick twice". ${ }^{3}$

It is worth noting that these two simple Bayesian surprise models have a number of shortcomings. They neither grasp the complexity of some magic tricks nor correctly represent the observer's beliefs, which, for example, are not usually explicitly estimating the magician's skills in probabilistic terms. Such models need to be tested, validated and optimized against behaviour, and a variety of additional variables need to be added to the models to better represent the effect of magic, such as an individual levels of suspicion as a bias towards either the prior (if the viewer does not trust the observations) or towards posterior (if the viewer is susceptible to magic). Moreover, the general setting of viewing a magic trick is likely to alter priors, and the behaviour and confidence of the magician may influence to which extent an "anything is possible" attitude may emerge.

Another more general concern regarding the application of Bayesian surprise for modelling the effect of magic is that humans do not always behave like optimal Bayesian observers, because we show a variety of cognitive biases when judging in uncertainty, and that Bayesian models strongly depend on the parameters used (Bowers and Davis, 2012; Marcus and Davis, 2013). However, there is also strong evidence that human often behave like Bayesian observers (Griffiths and Tenenbaum, 2006; Tenenbaum et al., 2006; Griffiths et al., 2012), and that it is also possible to model such biases in case they play a role in the experience of magic, by, for example, implementing a forgetting factor to lower the impact of the prior (cf. Itti and Baldi, 2009).

Yet, leaving these concerns aside, the simple Bayesian models of surprise presented here do illustrate the possibility of an approximate quantification of the "wow" effect in magic and also reflect a central element of magic: its effect is always dependent on the viewers' expectation. Just by having different prior beliefs, the very same information can be far more surprising. And this is how magic works.

\subsection{Misdirection in magic as manipulation of priors}

We can now turn our attention to the preparation phase of a magic trick. How do magicians construct the right prior beliefs to their tricks? The answer most magicians would give is by using misdirection. But what is misdirection? And what is misdirection in the context of Bayesian inference?

Misdirection has been extensively investigated, described and classified in the past (e.g. Lamont and Wiseman, 1999; Kuhn et al., 2008, 2014; Macknik et al., 2008; Kuhn and Martinez, 2012; Quiroga, 2016), yet there is no real consensus about what this concept exactly describes. Here, we will follow a rather broad understanding of misdirection and describe it as "that which directs the audience towards the effect and away from the method" (Lamont and Wiseman, 1999, cf. Macknik et al., 2008 and Kuhn et al., 2014). As such, all of the different methods, objects and actions magicians' use during the preparation phase to separate between what the viewers believe is happening and what is really going on fall under the umbrella concept of misdirection. Thus, misdirection in a Bayesian framework can be understood as the whole process of manipulation of the viewer's beliefs away from the real cause of the magic effect (the method) and to attract them to alternative beliefs to maximize the surprise of a magic trick. Accordingly, one can think of misdirection as entailing almost everything of the preparation phase, in as much as it is a mean to the trick's end. In consequence, objects, gestures, movements, jokes, clothing, lights, sounds, location, scenography and so forth, can be elements of misdirection.

The purpose of misdirection is to maximize the surprise of a trick by generating a

\footnotetext{
${ }^{3}$ Note that some trick routines indeed involve the repetition of the same effect, sometimes even using the very same method, with at least a constant or even increasing effect. However, all these tricks either make sure that the prior beliefs of the observers is "reset" after each effect to the wanted one or rely on "cognitively impenetrable" illusions (Ekroll et al., 2018). In both cases, repetitions will evoke a large surprise, in line with the understanding of the magic effect as Bayesian surprise. Resetting the priors can be done in several different ways: by changing the method, by delivering a false solution, by patter (i.e. spoken presentation), and so forth. A nice example of this is the entertaining "Six card repeat" by Tommy Tucker, whereby he repeatedly brings the viewers back to the starting belief by patter. An updated magic guideline could read: "never repeat a trick without the viewer holding the necessary prior beliefs".
} 
mismatch (i.e. large prediction error) between the viewer's expectations (prior beliefs) and what they observe. To achieve this, the magician needs make sure that the viewer's mind is brought into the right mind-set and that the real causes underlying the magic effect are hidden. All elements of misdirection serve this purpose.

\subsubsection{Types of misdirection}

There are different ways in which misdirection serves its purpose and these are largely constrained by the idiosyncrasies of our Bayesian architecture. Here, we propose a theorydriven fundamental classification of misdirection based on how misdirection affects inference and, as consequence, surprise. A comparison of the theory-driven classification of misdirection presented here and elsewhere goes beyond the scope of this paper. Nevertheless, it is worth noting that prior accounts are not in conflict with the Bayesian one presented in the following. We refer to the paper by Kuhn and colleagues for an extensive review on further classifications of misdirection (Kuhn, Caffaratti, Teszka, \& Rensink, 2014).

Reflecting on a simplified form of the Bayes theorem, $P(M \mid D) \propto P(D \mid M) P(M)$, it is evident that there are only two ways of directly affecting inference: by modifying the prior $P(M)$ or by modifying the likelihood $P(D \mid M)$. Purposely modifying either of these can help increase the Kullback-Leibler distance (surprise) between the prior $P(M)$ and posterior $P(M \mid D)$. Thus, misdirection can be used to maximize the surprise by either shifting the expectations or by shifting the sensory information (Figure 2). Most, if not all, magic tricks usually rely on a combination of both approaches. Magic tricks in which the effect is evoked using familiar sensory information (e.g. seeing a coin in an open hand) will need to modify the priors using misdirection (e.g. by making the viewer believe that the hand is empty) (Figure 2A). In turn, magic tricks in which the effect is evoked by the exploitation of deeply held (hyper-) priors about the world (e.g. massive objects on earth experience gravitation) will need to rely on the presented sensory information to evoke surprise (e.g. by presenting a seemingly levitating chair) (Figure 2B).

Finally, magic tricks use attentional control to modify the precision of prediction errors in favour of those that will ultimately maximize the distance between prior and posterior during the effect. Or, put in simpler terms, magicians basically control towards what, where and when the viewers should pay attention in order to maximize the surprise. Importantly, there are two components to attentional control: first, attention is directed such that the method is not discovered (e.g. to prevent the viewer from detecting the strings from which the chair hangs) (Figure $2 \mathrm{C}$ ). Second, attention is drawn to cognitive and sensory evidence that supports the trick. This increase in the observers' confidence in having observed the relevant information sharpens the probability distributions, hence increasing the surprise effect. Taken together, we propose three types of misdirection based on their distinct role in Bayesian inference:

1) Misdirection that uses evidence to generate new beliefs.

2) Misdirection that uses evidence to exploit existing (hyper-) priors.

3) Misdirection that filters the incoming information via attentional control.

All three distinct types of misdirection are, however, closely related and often intertwined. The first two types of misdirection can be understood as "setting the scene" and "exploiting the scene". That is, they construct and exploit a background context to aid the selection and generation of wanted priors to achieve a final mind-set in which the effect occurs. New evidence can be used to (1) create new beliefs ("The hand is empty") and/or to (2) increase the saliency of pre-existing beliefs ("Objects do not appear just like that") and so construct the wanted context in which the magic effect occurs ("Wow! Where does this coin comes from?!"). In turn, attentional control (3) filters and weights the incoming evidence with the aim of biasing inference via precision weighting away from the method. Attentional control mechanisms (3) are ultimately used for the generation (1) and/or exploitation (2) of background beliefs via effective filtering of the incoming evidence.

Importantly, the three types misdirection used to maximize surprise can occur at very different levels of the cortical hierarchy. This depends on which beliefs are generated or targeted and which attentional mechanism is manipulated. Below, we will present examples of the three types of misdirection in the context of Bayesian inference. Please note that most of these misdirection methods and examples have been described elsewhere in greater detail 
(Lamont and Wiseman, 1999; Kuhn et al., 2008, 2014; Macknik et al., 2008; Kuhn and Martinez, 2012; Quiroga, 2016).

\section{A. Creation of new expectations}

Generation of priors away from data (e.g. when input is familiar)

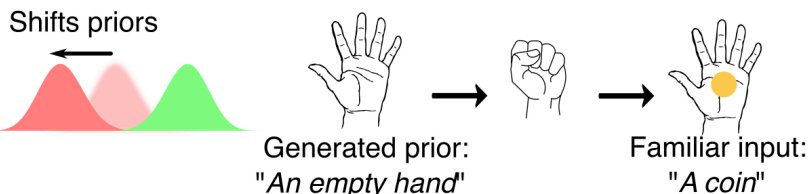

\section{B. Exploitation of prior knowledge}

Present data that violates existing priors (e.g. like gravitation)

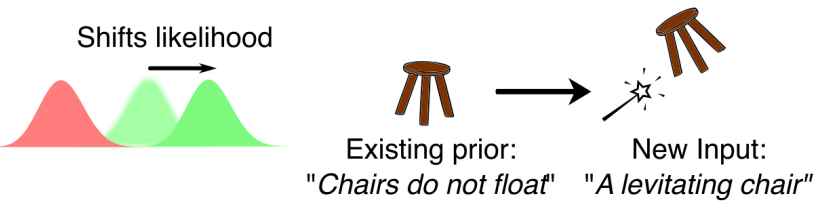

\section{Attentional control}

Selection and weightening of data (e.g. when method are visible)

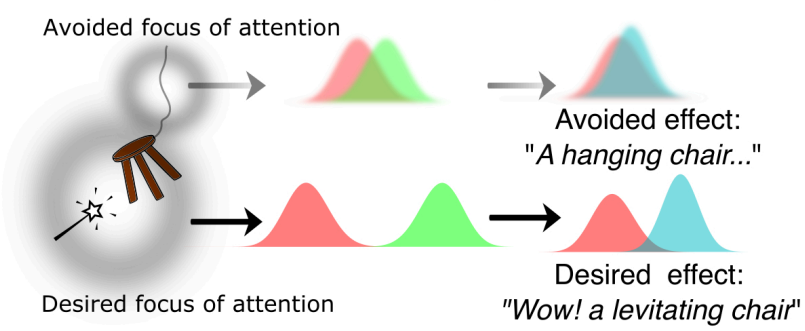

Figure 2. Types of misdirection and their effects on probability distributions of sensory input and expectation. A. The generation of new expectations is used to shift prior beliefs (red distribution) away from the sensory information (green distribution) to, as a consequence, increase surprise. This form of misdirection is often used when the effect of the trick relies on familiar and mundane sensory information, like a coin. Magicians then create specific beliefs in which context the otherwise uninteresting sensory information elicits surprise. B. Exploitation of prior knowledge is always present in magic. However, some tricks rely almost exclusively on this. In such tricks, magicians will need to present sensory information that violates their already existing prior knowledge about the world. This will increase the distance between both distributions by shifting the likelihood, and hence amplify surprise. Examples of this principle are levitation tricks, as shown with a levitation chair. Here, magicians exploit our prior knowledge of gravitational forces to create surprising effects. C. The third type of misdirection is attentional control. In this case, magicians do not directly intervene with the prior or likelihood but bias the selection and weighting of these distributions (at different levels of the cortical hierarchy). For example, magicians will try to direct attention away from the method, because this would destroy the effect. Instead, magicians will bring the audience to focus on sensory information that maximizes surprise. This is depicted in the figure as the levitation chair (desired focus of attention) and the string from which the chair is hanging (avoided focus of attention).

\subsubsection{Example of misdirection based on creating new priors: Repetition}

One of the most common misdirection techniques used to generate novel prior beliefs is repetition. For example, by repeating movements, magicians shape the expectation of the movement to be repeated again. By stopping or changing the movement the magician can induce a strong effect in the viewers. This is probably one of the simplest and yet extremely effective ways of generating surprise. Kuhn and colleagues investigated in a series of eyetracking studies a very simple trick (the "Vanishing Ball Illusion") that implements this form of misdirection (Kuhn and Land, 2006; Kuhn and Rensink, 2016). The trick consists of the magician throwing a ball upwards twice and the third time pretending to throw the ball while secretly keeping it in the hand. Strikingly, a large amount of the participants claimed to have seen ball leave his hand and disappear in the air (Kuhn and Land, 2006). Here, Kuhn generates an expectation in the viewers' mind based on repetitive movements and then simply breaks it, inducing thereby the perception of an illusory ball that surprisingly vanishes mid-air in most of the participants. If, however, participants were not shown the real throws before the deceptive throw (i.e. without the generation of a prior), the probability of experiencing the illusion was reduced to the half (Kuhn and Rensink, 2016), in line with the understanding of the magic effect as Bayesian surprise. 


\subsubsection{Example of misdirection based on exploiting existing priors: Visual illusions}

An excellent example of magicians exploiting already existing perceptual (hyper-) priors to evoke surprise is the use of visual illusions. By exploiting simple perceptual organizational principles, such as amodal completion (Ekroll et al., 2013, 2016), amodal absence (Ekroll et al., 2017) or the Gestalt rule of "good continuation" magicians can create surprising effects such as the "cut-and-restore" rope trick (Barnhart, 2010). The essence of magic tricks exploiting such visual perceptual priors is to create an objectively ambiguous sensory situation, in which the most likely interpretation of it is also the wrong one. Figure 3 shows two well-known examples of this principle.

A.

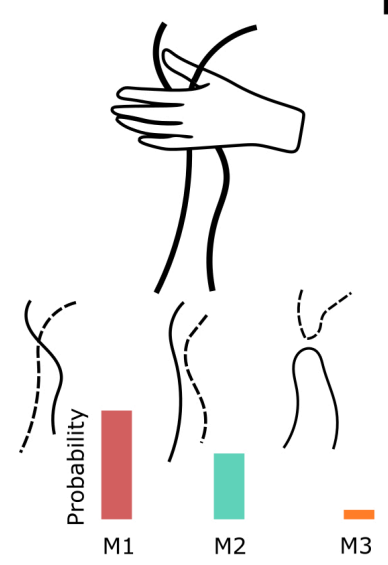

B.

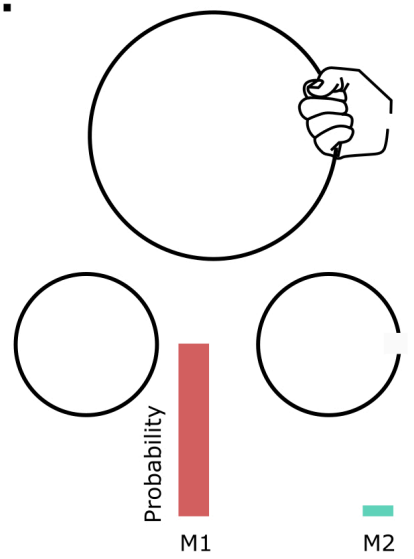

Figure 3. A. In the "cut-and-restore" trick a rope is seemingly cut in the middle. However, using a sleight-of-hand the magician cut the rope close to the edge, making two ropes of unequal length. In view of the ambiguous information (i.e. the hand occluding part of the ropes) the most probable percepts are two long ropes either crossing (M1) or not crossing (M2) behind the hand because of the Gestalt rule of good continuation and prior knowledge (i.e. the magician cut the rope seemingly in the middle). The perception (perceptual belief) of two ropes of unequal lengths behind the hand (M3) is far less probable (as illustrated by the coloured bars) and yet, true. B. Some magic tricks involving metal rings consist of seemingly closed rings, which have in fact a small opening. Covering this gap creates the impression in the viewer's mind that these are closed rings (M1). In contrast, the interpretation of this ambiguous information as a ring with a small gap is far less likely (M2).

\subsubsection{Examples of misdirection based on attentional control}

Besides the manipulation of priors, another central form of misdirection is attentional control. Magicians modify the selection and precision of the incoming information across time and space using attentional control. For simplicity, we can separate here between saliencydependent (bottom-up) and goal-directed (top-down) attentional control (Corbetta and Shulman, 2002). For a detailed analysis of different forms of attentional misdirection we refer to the paper by Kuhn and colleagues (Kuhn et al., 2014).

Saliency-dependent techniques are those that involve the use of salient stimuli (of whatever modality) to capture the viewer's attention to a specific location, object, moment or movement. These techniques are effective and robust because attention-capturing stimuli make viewers essentially "blind" for everything else. The list of examples of this form of misdirection is extensive: from a simple snapping of fingers to the production of a rabbit.

Probably the most often used form of saliency-based attentional control in close-up magic is hand movement. The magician's wisdom "a big move covers a small move" is a good depiction of this. If more than one movement are simultaneously visible, by capturing bottom-up attention of the viewer by means of a large, salient movement, magicians can cover a smaller (secret) move (Macknik et al., 2008). In Bayesian predictive coding terms, this corresponds to an increase of the precision weights of the large movement while suppressing the precision of the prediction error units representing the small movement. Thus, even if the small movement is visually processed, because of the large movement capturing our attention, the prediction errors of the small movement are going to be weighted as "noisy" and, as a consequence, ignored (see as an example Kuhn and Tatler, 2004).

Beyond the large repertoire of multimodal saliency-dependent attentional capturing techniques, magicians also apply several "top-down" attentional control techniques. The 
easiest and simplest way of capturing attention in a goal-directed top-down manner is just by explicitly asking the viewers to pay attention to a particular thing or to do some task (like writing something down). This can be done to divert the viewers' attention from, for example, a secret action, or to make them focus on something important that supports a prior set of beliefs necessary for the trick (Lamont and Wiseman, 1999). For example, card magicians may subtly ask the viewers to pay attention onto how they shuffle the cards to aid the generation of the belief that the cards are in random order, while performing a false shuffle (which are techniques that appear to fairly shuffle a deck, when actually the cards in the deck are controlled to maintain a specific order). By doing this, the magicians increase the probability that the viewers believe that the deck is shuffled.

\subsubsection{Further interesting examples of misdirection}

An often-used form of misdirection that exploits prior knowledge is contextual information. Purposely used contextual information can bring the viewers into establishing a specific context and thus, favouring certain existing priors. This is best illustrated by the many magic tricks that involve mundane, well-known objects (like cards, coins, bills and ropes) because they are familiar, do not raise suspicion and evoke clear expectations. Similarly, contextual information helps to understand why life magic shows are more surprising than recorded ones (because they could be manipulated) and why we are only surprised about magic in the real world and not in fictional contexts like in The Lord of the Rings or Harry Potter (because our general knowledge of the world does not hold in fictional worlds).

Along the same lines, another example of contextual misdirection is close-up magic taking place in everyday scenarios like the street, supermarket or shop without people knowing they are actually witnessing a magician. An entertaining example of how this contextual misdirection can maximize the effect of magic are the tricks on unsuspecting people performed by the magician Michael Carbonaro in his hidden-camera show "The Carbonaro Effect". His tricks target completely random people and evoke extreme reactions to "impossible" events, like seeing a woman turn into a mermaid or a baby dinosaur hatching from an egg. Key to this form of misdirection is that it negates the natural suspicion observers have when knowing they are about to witness a magic trick.

Yet another example of contextual misdirection are the background stories magicians often use during their tricks. These background stories can have a deep effect in how the magic effect is experienced. For example, framing a simple conjuring trick in terms of genuine psychic abilities can affect the interpretation of the magic event. Participants told that the trick is done by a conjuror or psychic, respectively, later explained the trick in terms of conjuring abilities or of psychic abilities, respectively (Mohr et al., 2015).

Finally, fascinating examples of misdirection generating novel cognitive beliefs are so-called "false solutions" (Tamariz, 1988). Magicians use false solutions in "sucker effects" in which the viewers are led to believe that they have detected or been told the method of a trick, but then the magician reveals that this is not the case. Accordingly, false solutions are used to "explain" the method behind their tricks (e.g. saying that all cards in the deck are the same) with the aim to divert the viewers from the real method of the trick (e.g. they forced a card to the viewer) and maximize the surprise.

Previous research with chess experts showed that the existence of a familiar solution to a (chess) problem can prevent the participants to find a less familiar, yet more efficient solution (Bilalić et al., 2008b, 2008a, 2010). In such cases, the subjects are "fixed" to the more salient solution and are unlikely to find the alternative ones. This cognitive bias is called the "Einstellungs effect" and is often exploited by magicians in their performances. In contrast to the chess results though, viewers in magic are also often fooled into believing unlikely or even physically impossible solutions. A recent study investigated this effect using a card trick with an extremely simple method (Thomas and Didierjean, 2016). They showed that $83 \%$ of the participants could find the solution of the trick without any problem. However, if participants were presented a false solution at the beginning of the trick, only $17 \%$ of the participants found the correct solution, even though the false solution was unlikely and unfamiliar (they were told that their card choice was going to be influenced by a hand movement of the magician). This shows the effectiveness of false solutions: a single exposure to an unlikely and unfamiliar false solution can prevent participants from finding the more obvious simpler solution (all cards were the same), even if encouraged to look for alternatives. 


\subsection{Some final notes on misdirection}

In sum, we hope to have shown how misdirection in magic can be understood as a procedure maximizing the "wow" effect. Specifically, this is achieved via the selective manipulation of information to (1) create new specific beliefs, to (2) exploit previously existing knowledge, and (3) direct the viewers' attention away from secret methods behind the magic trick and towards selective information, thus also affecting precision estimates of sensory information.

An important point of misdirection is that its success relies upon the observers not noticing that they are being misdirected. Indeed, effective misdirection is not about crude distraction (Lamont and Wiseman, 1999). Viewers need to trust the perceived information and have the impression they saw everything that was to be seen. Hence, magicians try to avoid "noisy" presentations, so that the viewers can trust their perception and think of them as being precise and reliable, up to the moment of magical surprise. Only attended and well-perceived objects and actions receive a precise and reliable probability distribution, and correspondingly, this results in larger surprise when the expectation is violated. Clearly, the appearance of a person or an object in a noisy situation, such as stage full of smoke, with the lights off or cluttered with objects, is not as surprising as its appearance in a well-illuminated stage without further elements.

In line with this, magicians avoid fast, suspicious movements, and try to make their actions look as "natural" as possible (Lamont and Wiseman, 1999). One of the first things card magicians learn is to make their sleight of hands appear natural (i.e. less salient)(Cavina-Pratesi et al., 2011; Phillips et al., 2015), to avoid hasty movements while performing them and only to perform a card trick if the sleight of hand is controlled and smooth. By doing so, they ensure two things: first, that the spectators do not detect the sleight of hand (Phillips et al., 2015) and second, it maximizes the achieved surprise during the magical effect by avoiding giving possible explanations, and by providing the viewers with "reliable" information. Hasty unnatural movements might allow the spectators to notice that "something just happened" even if they might not know what exactly happened, which might be enough to ruin the trick by reducing the surprise of the magical effect.

Moreover, to maximize the "wow" effect and to avoid detection of the methods, it is often stressed that magicians should never repeat a magic trick using the same methods and should try to avoid the viewers getting into a "problem-solving" mode, which is often taken after repeated presentation of a magic trick (Danek et al., 2014b, 2014a). Indeed, empirical studies show that viewers are less misdirected if they observe the same trick twice (Kuhn and Tatler, 2004). Often, it is sufficient that the viewers just falsely believe that they can explain what they just observed in order to destroy the magic.

\section{Conclusion and future directions}

We have presented here a first tentative understanding of magic within a Bayesian predictive coding framework. We argued that the "wow" effect magic tricks evoke is the phenomenological experience of a profound mismatch between the viewers' prior beliefs and what they perceive. We propose that this effect can be understood as Bayesian surprise, and that it can be computed as the difference between beliefs before and after an observation. We claim that magic tricks strive simply "to evoke surprise" (Figure 4A). Moreover, we present here a broadly defined concept of misdirection that describes it as all elements of the preparation phase that are means to maximize the surprise, i.e. the distance between prior and posterior beliefs and the sharpening of probability distributions (Figure 4B for an example). Specifically, we showed that misdirection can bias inference in three ways: (1) through the generation and (2) exploitation of prior beliefs and (3) via control of attentional resources to select and weight the incoming information. We believe that the understanding of magic within this Bayesian predictive coding framework presents manifold advantages that could be of aid for scientists in future investigations.

First and foremost, this framework aims to conceptualize the phenomenon of magic in simple and known terms, making it tractable, comparable and unifiable with other models and theories. This allows the study of magic to profit from the development of cognitive models (e.g. models of arousal, surprise, judgment in uncertainty, decision-making, attentional selection, belief-updating, etc.) and brings the results of the hitherto somewhat fringe science of magic closer to other research fields of cognitive neuroscience and psychology. Essential 
for this is the understanding of the effect of wonder and "impossibility" in magic "just" as the experience of surprise and not as a distinct mysterious phenomenological experience. This allows the integration of the experience of magic in unified model of perception and cognition.

Second, positioning the phenomenon of magic within a Bayesian framework also allows for a detailed categorization of different misdirection methods depending on which kind of prior belief they generate or exploit during the preparation phase to generate the effect (e.g. a visual prior, a cognitive prior, etc.). Thus, previous psychological approaches to taxonomize misdirection and tricks (Kuhn et al., 2014; Rensink \& Kuhn, 2015) can be easily reformulated in Bayesian predictive coding terms, albeit it is questionable of how meaningful this taxonomical approach might be (Lamont et al., 2010; Lamont, 2015).

Third, given the extensive previous work within the Bayesian predictive framework in different fields (e.g. neuroscience, psychology and psychiatry), it allows making testable hypotheses in each of them. Magic can be an interesting test field in which predictions from Bayesian predictive models can be tested in a highly robust, ecological, entertaining and naturalistic manner (cf. Macknik et al., 2008; Quiroga, 2016). Consider, just as an example, the predictive coding account of psychosis, which relates psychotic symptoms to a decreased impact of prior beliefs and/or an increased precision of the sensory data during Bayesian inference (Corlett et al., 2009, 2016; Sterzer et al., 2018). Given that inference is then strongly driven by the incoming information and prior beliefs play a weaker role, we expect patients showing psychotic symptoms to take magic tricks more or less at face value and elicit weaker surprise responses.

Finally, as Bayesian predictive coding approaches are computational models of brain function, they allow the modelling of magic effects and their empirical testing. As an example, we introduced here a computational understanding of (Bayesian) surprise and implemented it for two examples of simple Bayesian observer models of probabilistic tricks. These models of surprise were capable of capturing essential features of the effect of magic, namely the dependency of the "wow" effect based on prior beliefs and repetition suppression after multiple presentations. More complex hierarchical Bayesian models of surprise can be used to model the "wow" effect of a large variety of tricks and to correlate these model-based predictions with both, behavioural and physiological measurements. This latter approach seems specially promising in view that only a handful of studies investigated the physiological effect of magic (Parris et al., 2009; Danek et al., 2015; Caffaratti et al., 2016). Crucially, a number of physiological signatures have been already related specifically to Bayesian surprise, such as pupil dilation (Zénon, 2019), the P300 evoked related potential in EEG (Mars et al., 2008; Wacongne et al., 2011; Kolossa et al., 2012; Kopp et al., 2016) and fMRI responses in the dorsal anterior cingulate cortex (Ide et al., 2013; O'Reilly et al., 2013; Schwartenbeck et al., 2016). This previous empirical work and theoretical framework allows a variety of hypothesis-driven physiological studies of magic tricks, such as the parametric modulation of neural signals as a function of surprise, contextual information and attentional control.

In conclusion, we believe that positioning the phenomenon of magic within an already established and empirically tested framework of brain function as attempted here can be beneficial to both, the nascent field of the science of magic and cognitive neuroscience. 
A.

Bayesian Surprise:

$\mathrm{KL}$-divergence between beliefs

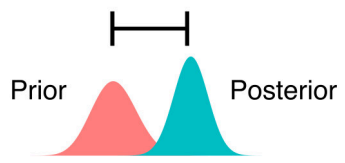

Bayesian brains aim: "Not to be surprised"
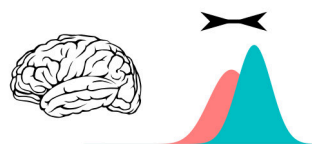

Magicians aim:

"To evoke surprise"

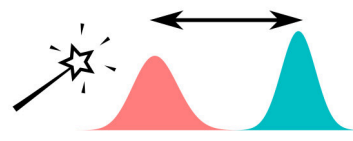

B.

\section{Magic: coin production}

Preparation phase

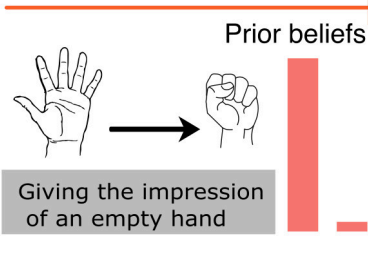

M1 M2
Effect phase

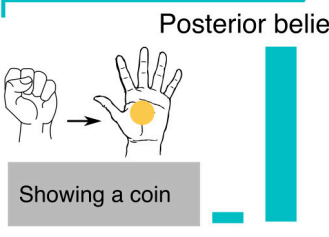

M1 M2

\section{Not magic: showing a coin}

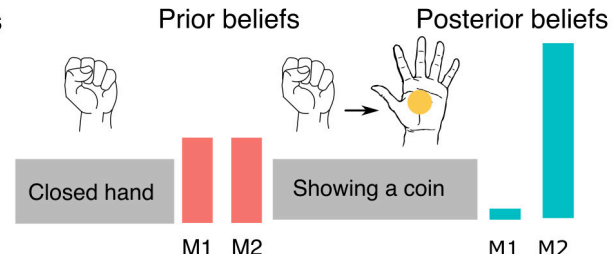

Surprise is low

"A coin? Ok..."
M1 M2

Beliefs:

Surprise is high

M1: "Empty hand"

M2: "No empty hand"

"Wow! Where does this coin comes from?"

Figure 4. A. Bayesian surprise is calculated as the Kullback-Leibler $(K L)$ divergence between beliefs before and after an observation. We propose to use this measurement to quantify the "wow" effect of magic. A Bayesian brain encountering a familiar situation will experience a minimal distance between the prior and incoming sensory data and, in consequence, posterior ("no surprise"). Magic strives to maximize the distance between beliefs before and after the culmination of a trick ("to evoke surprise"). B. An example of misdirection. On the left we see a coin production trick, on the right just someone showing a coin. Both situations have the same sensory information: a hand is opened to reveal a coin. However, the sensory information only elicits surprise if the prior belief "empty hand" (M1) was generated during the preparation phase of the trick by showing that the hand is apparently empty. In contrast, when just seeing a closed hand, the belief "empty hand" (M1) and "no empty hand" (M2) are both equally likely, so that the surprise by seeing a coin is low. 


\section{Conflict of interest}

The authors declare no conflict of interest.

\section{Author contributions}

PRG: conception, design and drafting of the manuscript. AB: revision and supervision of the manuscript.

\section{Acknowledgements}

We thank Michael Bannert, Nicola Clayton and Clive Wilkins for vivid discussions about the ideas presented in this manuscript and comments on a previous draft of this manuscript.

\section{References}

Alexander, W. H., and Brown, J. W. (2011). Medial prefrontal cortex as an action-outcome predictor. Nat. Neurosci. 14, 1338-1344. doi:10.1038/nn.2921.

Alexander, W. H., and Brown, J. W. (2019). The Role of the Anterior Cingulate Cortex in Prediction Error and Signaling Surprise. Top. Cogn. Sci. 11, 119-135. doi:10.1111/tops.12307.

Baldi, P., and Itti, L. (2010). Of bits and wows: A Bayesian theory of surprise with applications to attention. Neural Networks 23, 649-666. doi:10.1016/j.neunet.2009.12.007.

Bar, M. (2009). The proactive brain: Memory for predictions. Philos. Trans. R. Soc. B Biol. Sci. 364, 1235-1243. doi:10.1098/rstb.2008.0310.

Barnhart, A. S. (2010). The exploitation of Gestalt principles by magicians. Perception 39, 1286-1289. doi:10.1068/p6766.

Barnhart, A. S., and Goldinger, S. D. (2014). Blinded by magic: Eye-movements reveal the misdirection of attention. Front. Psychol. 5, 1-9. doi:10.3389/fpsyg.2014.01461.

Barto, A., Mirolli, M., and Baldassarre, G. (2013). Novelty or Surprise? Front. Psychol. 4, 1-15. doi:10.3389/fpsyg.2013.00907.

Bilalić, M., McLeod, P., and Gobet, F. (2008a). Inflexibility of experts-Reality or myth? Quantifying the Einstellung effect in chess masters. Cogn. Psychol. 56, 73-102. doi:10.1016/j.cogpsych.2007.02.001.

Bilalić, M., McLeod, P., and Gobet, F. (2008b). Why good thoughts block better ones: The mechanism of the pernicious Einstellung (set) effect. Cognition 108, 652-661. doi:10.1016/j.cognition.2008.05.005.

Bilalić, M., McLeod, P., and Gobet, F. (2010). The mechanism of the einstellung (set) effect: A pervasive source of cognitive bias. Curr. Dir. Psychol. Sci. 19, 111-115. doi:10.1177/0963721410363571.

Bowers, J. S., and Davis, C. J. (2012). Bayesian Just-So Stories in Psychology and Neuroscience. 138, 389-414. doi:10.1037/a0026450.

Caffaratti, H., Navajas, J., Rey, H. G., and Quian Quiroga, R. (2016). Where is the ball? Behavioral and neural responses elicited by a magic trick. Psychophysiology 53, 1441-1448. doi:10.1111/psyp.12691.

Cavina-Pratesi, C., Kuhn, G., letswaart, M., and da Milner, A. D. (2011). The magic grasp: Motor expertise in deception. PLoS One 6. doi:10.1371/journal.pone.0016568.

Chun, M. M. (2000). Contextual cueing of visual attention. Trends Cogn. Sci. 4, 170-178. doi:10.1016/S13646613(00)01476-5.

Clark, A. (2013). Whatever next? Predictive brains, situated agents, and the future of cognitive science. Behav. Brain Sci. 36, 181-204. doi:10.1017/S0140525X12000477.

Clark, A. (2018). A nice surprise? Predictive processing and the active pursuit of novelty. Phenomenol. Cogn. Sci. 17, 521-534. doi:10.1007/s11097-017-9525-z.

Corbetta, M., and Shulman, G. L. (2002). Control of goal-directed and stimulus-driven attention in the brain. Nat. Rev. Neurosci. 3, 201-15. doi:10.1038/nrn755.

Corlett, P. R., Aitken, M. R. F., Dickinson, A., Shanks, D. R., Honey, G. D., Honey, R. A. E., et al. (2004). Prediction error during retrospective revaluation of causal associations in humans: fMRI evidence in favor of an associative model of learning. Neuron 44, 877-888. doi:10.1016/j.neuron.2004.11.022.

Corlett, P. R., Frith, C. D., and Fletcher, P. C. (2009). From drugs to deprivation: a Bayesian framework for understanding models of psychosis. 515-530. doi:10.1007/s00213-009-1561-0.

Corlett, P. R., Honey, G. D., and Fletcher, P. C. (2016). Prediction error, ketamine and psychosis: An updated model. J. Psychopharmacol. 30, 1145-1155. doi:10.1177/0269881116650087.

Cui, J., Otero-Millan, J., Macknik, S. L., King, M., and Martinez-Conde, S. (2011). Social Misdirection Fails to Enhance a Magic Illusion. Front. Hum. Neurosci. 5, 1-11. doi:10.3389/fnhum.2011.00103.

Danek, A. H., Fraps, T., von Müller, A., Grothe, B., and Öllinger, M. (2014a). It's a kind of magic-what self-reports can reveal about the phenomenology of insight problem solving. Front. Psychol. 5, 1-11. doi:10.3389/fpsyg.2014.01408

Danek, A. H., Fraps, T., von Müller, A., Grothe, B., and Öllinger, M. (2014b). Working Wonders? Investigating insight with magic tricks. Cognition 130, 174-185. doi:10.1016/j.cognition.2013.11.003.

Danek, A. H., Öllinger, M., Fraps, T., Grothe, B., and Flanagin, V. L. (2015). An fMRI investigation of expectation violation in magic tricks. Front. Psychol. 6, 1-11. doi:10.3389/fpsyg.2015.00084.

Egner, T., Monti, J. M., and Summerfield, C. (2010). Expectation and surprise determine neural population responses in the ventral visual stream. J. Neurosci. 30, 16601-16608. doi:10.1523/JNEUROSCI.2770-10.2010. 
Ekroll, V., De Bruyckere, E., Vanwezemael, L., and Wagemans, J. (2018). Never Repeat the Same Trick TwiceUnless it is Cognitively Impenetrable. Iperception. 9. doi:10.1177/2041669518816711.

Ekroll, V., Sayim, B., Van Der Hallen, R., and Wagemans, J. (2016). Illusory Visual Completion of an Object's Invisible Backside Can Make Your Finger Feel Shorter. Curr. Biol. 26, 1029-1033. doi:10.1016/j.cub.2016.02.001.

Ekroll, V., Sayim, B., and Wagemans, J. (2013). Against better knowledge: The magical force of amodal volume completion. Iperception. 4, 511-5. doi:10.1068/i0622sas.

Ekroll, V., Sayim, B., and Wagemans, J. (2017). The Other Side of Magic: The Psychology of Perceiving Hidden Things. Perspect. Psychol. Sci. 12, 91-106. doi:10.1177/1745691616654676.

Felleman, D. J., and Van Essen, D. C. (1991). Distributed hierachical processing in the primate cerebral cortex. Cereb. Cortex 1, 1-47. doi:10.1093/cercor/1.1.1.

Fletcher, P. C., Anderson, J. M., Shanks, D. R., Honey, R., Carpenter, T. A., Donovan, T., et al. (2001). Responses of human frontal cortex to surprising events are predicted by formal associative learning theory. Nat. Neurosci. 4, 1043-1048. doi:10.1038/nn733.

Friston, K. (2005). A theory of cortical responses. Philos. Trans. R. Soc. Lond. B. Biol. Sci. 360, 815-36. doi:10.1098/rstb.2005.1622.

Friston, K., and Kiebel, S. (2009). Predictive coding under the free-energy principle. Philos. Trans. R. Soc. Lond. B. Biol. Sci. 364, 1211-21. doi:10.1098/rstb.2008.0300.

Garcia-pelegrin, B. E., Alexandra, K., Wilkins, C., and Clayton, N. S. (2020). Experiments with magic effects might be informative about cognition in animals. Science 369, 1424-1426.

Griffiths, T. L., Chater, N., Norris, D., and Pouget, A. (2012). How the Bayesians Got Their Beliefs ( and What Those Beliefs Actually Are ): Comment on Bowers and Davis ( 2012 ). 138, 415-422. doi:10.1037/a0026884.

Griffiths, T. L., and Tenenbaum, J. B. (2006). Optimal Predictions in Everyday Cognition. 17, 767-773.

Harrison, L. M., Stephan, K. E., Rees, G., and Friston, K. J. (2007). Extra-classical receptive field effects measured in striate cortex with fMRI. Neuroimage 34, 1199-1208. doi:10.1016/j.neuroimage.2006.10.017.

Hohwy, J. (2012). Attention and conscious perception in the hypothesis testing brain. Front. Psychol. 3, 1-14. doi:10.3389/fpsyg.2012.00096.

Ide, J. S., Shenoy, P., Yu, A. J., and Li, C. S. R. (2013). Bayesian prediction and evaluation in the anterior cingulate cortex. J. Neurosci. 33, 2039-2047. doi:10.1523/JNEUROSCI.2201-12.2013.

Itti, L., and Baldi, P. (2005). Bayesian surprise attracts human attention. Adv. Neural Inf. Process. Syst., 547-554.

Itti, L., and Baldi, P. (2009). Bayesian surprise attracts human attention. Vision Res. 49, 1295-1306. doi:10.1016/j.visres.2008.09.007.

Kersten, D., Mamassian, P., and Yuille, A. (2004). Object perception as Bayesian inference. Annu. Rev. Psychol. 55, 271-304. doi:10.1146/annurev.psych.55.090902.142005.

Kersten, D., and Yuille, A. (2003). Bayesian models of object perception. Curr. Opin. Neurobiol. 13, 150-158. doi:10.1016/S0959-4388(03)00042-4.

Kolossa, A., Fingscheidt, T., Wessel, K., and Kopp, B. (2012). A model-based approach to trial-by-trial P300 amplitude fluctuations. Front. Hum. Neurosci. 6, 1-28. doi:10.3389/fnhum.2012.00359.

Kopp, B., Seer, C., Lange, F., Kluytmans, A., Kolossa, A., Fingscheidt, T., et al. (2016). P300 amplitude variations, prior probabilities, and likelihoods: A Bayesian ERP study. Cogn. Affect. Behav. Neurosci. 16, 911-928. doi:10.3758/s13415-016-0442-3.

Kuhn, G., Amlani, A. A., and Rensink, R. A. (2008). Towards a science of magic. Trends Cogn. Sci. 12, 349-354. doi:10.1016/j.tics.2008.05.008.

Kuhn, G., Caffaratti, H. A., Teszka, R., and Rensink, R. A. (2014). A psychologically-based taxonomy of misdirection. Front. Psychol. 5, 1-14. doi:10.3389/fpsyg.2014.01392.

Kuhn, G., and Land, M. F. (2006). There's more to magic than meets the eye. Curr. Biol. 16, 950-951. doi:10.1016/j.cub.2006.10.012.

Kuhn, G., and Martinez, L. M. (2012). Misdirection - Past, Present, and the Future. Front. Hum. Neurosci. 5, 1-7. doi:10.3389/fnhum.2011.00172.

Kuhn, G., and Rensink, R. A. (2016). The Vanishing Ball Illusion: A new perspective on the perception of dynamic events. Cognition 148, 64-70. doi:10.1016/j.cognition.2015.12.003.

Kuhn, G., and Tatler, B. W. (2004). Magic and fixation: Now you don't see it, now you do. 1155-1161.

Kveraga, K., Boshyan, J., and Bar, M. (2007). Magnocellular projections as the trigger of top-down facilitation in recognition. J. Neurosci. 27, 13232-13240. doi:10.1523/JNEUROSCI.3481-07.2007.

Lamont, P. (2015). Problems with the mapping of magic tricks. Front. Psychol. 6, 6-8. doi:10.3389/fpsyg.2015.00855.

Lamont, P. (2017). A Particular Kind of Wonder: The Experience of Magic past and Present. Rev. Gen. Psychol. 21, 1-8. doi:10.1037/gpr0000095.

Lamont, P., Henderson, J. M., and Smith, T. J. (2010). Where Science and Magic Meet: The Illusion of a "Science of Magic." Rev. Gen. Psychol. 14, 16-21. doi:10.1037/a0017157.

Lee, M. D. (2008). Three case studies in the Bayesian analysis of cognitive models. Psychon. Bull. Rev. 15, 1-15. doi:10.3758/PBR.15.1.1.

Lee, M. D., and Wagenmakers, E. J. (2013). Bayesian cognitive modeling: A practical course. Cambridge University Press doi:10.1017/CBO9781139087759.

Linden, D. E. J. (2005). The P300: Where in the brain is it produced and what does it tell us? Neuroscientist 11, 563576. doi:10.1177/1073858405280524.

Macknik, S. L., King, M., Randi, J., Robbins, A., Teller, Thompson, J., et al. (2008). Attention and awareness in stage magic: Turning tricks into research. Nat. Rev. Neurosci. 9, 871-879. doi:10.1038/nrn2473.

Marcus, G. F., and Davis, E. (2013). How Robust Are Probabilistic Models of Higher-Level Cognition ? 1-10. doi:10.1177/0956797613495418.

Mars, R. B., Debener, S., Gladwin, T. E., Harrison, L. M., Haggard, P., Rothwell, J. C., et al. (2008). Trial-by-trial fluctuations in the event-related electroencephalogram reflect dynamic changes in the degree of surprise. $J$. Neurosci. 28, 12539-12545. doi:10.1523/JNEUROSCI.2925-08.2008.

Mayrhauser, L., Bergmann, J., Crone, J., and Kronbichler, M. (2014). Neural repetition suppression: evidence for perceptual expectation in object-selective regions. Front. Hum. Neurosci. 8, 1-8. doi:10.3389/fnhum.2014.00225. 
Mohr, C., Koutrakis, N., and Kuhn, G. (2015). Priming psychic and conjuring abilities of a magic demonstration influences event interpretation and random number generation biases. Front. Psychol. 5, 1-8. doi:10.3389/fpsyg.2014.01542.

Mumford, D. (1992). On the computational architecture of the neocortex - II. The role of the corticocortical loops. Biol. Cybern. 66, 241-251. doi:10.1007/BF00202389.

O'Reilly, J. X., Schüffelgen, U., Cuell, S. F., Behrens, T. E. J., Mars, R. B., and Rushworth, M. F. S. (2013). Dissociable effects of surprise and model update in parietal and anterior cingulate cortex. Proc. Natl. Acad. Sci. U. S. A. 110. doi:10.1073/pnas.1305373110.

Parris, B. A., Kuhn, G., Mizon, G. A., Benattayallah, A., and Hodgson, T. L. (2009). Imaging the impossible: An fMRI study of impossible causal relationships in magic tricks. Neuroimage 45, 1033-1039. doi:10.1016/j.neuroimage.2008.12.036.

Phillips, F., Natter, M. B., and Egan, E. J. L. (2015). Magically deceptive biological motion - The French drop sleight. Front. Psychol. 6, 1-10. doi:10.3389/fpsyg.2015.00371.

Polich, J. (2007). Updating P300: An integrative theory of P3a and P3b. Clin. Neurophysiol. 118, $2128-2148$. doi:10.1016/j.clinph.2007.04.019.

Quiroga, R. Q. (2016). Magic and cognitive neuroscience. Curr. Biol. 26, R390-R394. doi:10.1016/j.cub.2016.03.061.

Rao, R. P., and Ballard, D. H. (1999). Predictive coding in the visual cortex: a functional interpretation of some extraclassical receptive-field effects. Nat. Neurosci. 2, 79-87. doi:10.1038/4580.

Rensink, R. A., and Kuhn, G. (2015a). A framework for using magic to study the mind. Front. Psychol. 6, 1-14. doi:10.3389/fpsyg.2015.01508.

Rensink, R. A., and Kuhn, G. (2015b). The possibility of a science of magic. Front. Psychol. 6, 1-3. doi:10.3389/fpsyg.2015.01576.

Schultz, W., Dayan, P., and Montague, P. R. (1997). A neural substrate of prediction and reward. Science 275, 1593-1599. doi:10.1126/science.275.5306.1593.

Schwartenbeck, P., Fitzgerald, T., Dolan, R. J., and Friston, K. (2013). Exploration, novelty, surprise, and free energy minimization. Front. Psychol. 4, 710. doi:10.3389/fpsyg.2013.00710.

Schwartenbeck, P., FitzGerald, T. H. B., and Dolan, R. (2016). Neural signals encoding shifts in beliefs. Neuroimage 125, 578-586. doi:10.1016/j.neuroimage.2015.10.067.

Smith, W., Dignum, F., and Sonenberg, L. (2016). The construction of impossibility: A logic-based analysis of conjuring tricks. Front. Psychol. 7, 1-17. doi:10.3389/fpsyg.2016.00748.

Sterzer, P., Adams, R. A., Fletcher, P., Frith, C., Lawrie, S. M., Muckli, L., et al. (2018). The Predictive Coding Account of Psychosis. Biol. Psychiatry 84, 634-643. doi:10.1016/j.biopsych.2018.05.015.

Summerfield, C., Trittschuh, E. H., Monti, J. M., Mesulam, M.-M., and Egner, T. (2008). Neural repetition suppression reflects fulfilled perceptual expectations. Nat. Neurosci. 11, 1004-1006. doi:10.1038/nn.2163.

Tamariz, J. (1988). The Magic Way. Madrid: Editorial Frankson Magic Books.no

Tenenbaum, J. B., Griffiths, T. L., and Kemp, C. (2006). Theory-based Bayesian models of inductive learning and reasoning. 10. doi:10.1016/j.tics.2006.05.009.

Thomas, C., and Didierjean, A. (2016). Magicians fix your mind: How unlikely solutions block obvious ones. Cognition 154, 169-173. doi:10.1016/j.cognition.2016.06.002.

Thomas, C., Didierjean, A., and Kuhn, G. (2018). It is magic! How impossible solutions prevent the discovery of obvious ones? Q. J. Exp. Psychol. 71, 2481-2487. doi:10.1177/1747021817743439.

Thomas, C., Didierjean, A., Maquestiaux, F., and Gygax, P. (2015). Does magic offer a cryptozoology ground for psychology? Rev. Gen. Psychol. 19, 117-128. doi:10.1037/gpr0000041.

Todorovic, A., van Ede, F., Maris, E., and de Lange, F. P. (2011). Prior expectation mediates neural adaptation to repeated sounds in the auditory cortex: An MEG study. J. Neurosci. 31, 9118-9123. doi:10.1523/JNEUROSCI.1425-11.2011.

Todorović, D. (2010). Context effects in visual perception and their explanations. Rev. Psychol. 17, 17-32. Available at: http://hrcak.srce.hr/70655?lang=en.

Turner, D. C., Aitken, M. R. F., Shanks, D. R., Sahakian, B. J., Robbins, T. W., Schwarzbauer, C., et al. (2004). The role of the lateral frontal cortex in causal associative learning: Exploring preventative and super-learning. Cereb. Cortex 14, 872-880. doi:10.1093/cercor/bhh046.

Utzerath, C., St. John-Saaltink, E., Buitelaar, J., and de Lange, F. P. (2017). Repetition suppression to objects is modulated by stimulus-specific expectations. Sci. Rep. 7, 8781. doi:10.1038/s41598-017-09374-z.

Van de Cruys, S., Wagemans, J., and Ekroll, V. (2015). The put-and-fetch ambiguity: How magicians exploit the principle of exclusive allocation of movements to intentions. Iperception. 6, 86-90. doi:10.1068/i0719sas.

Wacongne, C., Labyt, E., Van Wassenhove, V., Bekinschtein, T., Naccache, L., and Dehaene, S. (2011). Evidence for a hierarchy of predictions and prediction errors in human cortex. Proc. Natl. Acad. Sci. U. S. A. 108, 20754-20759. doi:10.1073/pnas.1117807108.

Zénon, A. (2019). Eye pupil signals information gain. Proc. R. Soc. B Biol. Sci. $286,0-1$. doi:10.1098/rspb.2019.1593. 